InnOvaciOnes de NegOciOs 17(34): 145-168

(C) 2020 UANL, Impreso en México (ISSN: 2007-1191)

Recepción: 6 de enero 2020 Aceptación: 22 de abril 2020

\title{
Desempeño financiero posterior de fusiones y adquisiciones de empresas públicas mexicanas (Financial performance after mergers and acquisitions of mexican public companies)
}

\author{
Viviana Lambretón Torres, Judith Villareal Escamilla, Ezequiel D. \\ Montemayor Garza, Juan P. Lechuga Elizondo, Laura A. Quiroga \\ Galindo \\ Universidad de Monterrey \\ viviana.lambreton@udem.edu
}

\begin{abstract}
Mergers and acquisitions have become a common strategy for business growth and consolidation, however, it has been found that in most cases this objective is not achieved since there is a lack of knowledge of the factors that affect post M\&A performance. This study aims to measure the change in financial metrics after 24 mergers and acquisitions in Mexico during 2013 and 2014. The Wilcoxon test is used to determine the significance of the change in subsequent performance. The results obtained show, in most of the financial ratios analyzed, a negative impact on subsequent financial performance and value creation. A robust regression model was used in order to identify some of the factors that cause their success or failure. An analysis of these factors could help to understand and implement corrective measures to achieve successful planning and implementation.
\end{abstract}

Key words: ratio analysis; mergers and acquisitions; pre and postperformance.

JEL: G140; G310; G320

Resumen: Las fusiones y adquisiciones se han vuelto una estrategia común para el crecimiento y consolidación de los negocios, sin embargo, se ha encontrado que en la mayoría de los casos no se logra este objetivo ya que existe un desconocimiento de los factores que inciden en el desempeño financiero posterior. Este estudio tiene como finalidad medir el cambio en métricas financieras posterior a 24 fusiones y adquisiciones llevadas a cabo en

\section{Desempeño financiero posterior de fusiones y adquisiciones}


México durante los años 2013 y 2014. Mediante la prueba de Wilcoxon se determina la significancia del cambio en el desempeño posterior. Los resultados obtenidos muestran, en la mayoría de las razones financieras analizadas, un impacto negativo en desempeño financiero posterior y en la creación de valor. Un modelo de regresión robusta fue utilizado con la finalidad de identificar algunos de los factores que ocasionan el éxito o fracaso de las mismas. Un análisis de dichos factores podría ayudar a comprender e implementar medidas correctivas que permitan lograr una planeación e implementación exitosa.

Palabras clave: análisis financiero; fusiones y adquisiciones; desempeño previo y posterior.

\section{Introducción}

Las fusiones y adquisiciones (FyA) son estrategias frecuentemente utilizadas por empresas de todo el mundo con la finalidad de alcanzar objetivos estratégicos, generar sinergias, obtener ventajas competitivas y crear valor para sus accionistas. Estas prácticas pueden impactar tanto positivamente como negativamente el desempeño financiero posterior de la empresa adquiriente.

Cuando se unen dos organizaciones con culturas, personalidades empresariales y sistemas de valores distintos, el éxito dependerá en gran medida en cómo estas organizaciones lleguen a integrarse. "Se considera que las fusiones y adquisiciones son convenientes, cuando el valor de la empresa combinada es mayor al valor de las dos empresas independientes consideradas", por lo tanto, cuando las empresas logran hacer una fusión 0 adquisición en la cual aumenta su valor esperado en conjunto, se logra obtener un efecto sinérgico, incrementando la rentabilidad y el valor de empresa, además del valor de los accionistas. (Carmona et al., 2015).

A través de las FyA se pueden generar sinergias operativas y financieras que deberían beneficiar a las compañías, sin embargo, de acuerdo a Damodaran (2005), "Los beneficios de las sinergias no se dan como deberían debido a adquisiciones incorrectamente valuadas y mal planeadas de forma tal que, lo que originalmente se planea en papel, no puede llevarse a cabo en la práctica." En opinión de Andrade et al., (2001) "Independientemente de la importancia de las fusiones y adquisiciones como una estrategia de

\section{Lambretón, T., Villarreal, J., Montemayor, E., Lechuga, J. \& Quiroga, L-}


crecimiento corporativo, entre el $60 \%$ y el $80 \%$ de las fusiones y adquisiciones no producen mayores ganancias".

De acuerdo al reporte de que presenta Seale \& Associates, en el año 2017 se reportaron 220 transacciones de FyA en México por un monto total de 21 mil millones de dólares, por lo que es importante determinar si esta estrategia empresarial es favorable o no para el crecimiento económico de las empresas mexicanas.

La mayoría de las investigaciones que se han realizado sobre FyA analizan el impacto del anuncio de la fusión en el precio y rendimiento anormal de las acciones de la compañía adquirida, como es el caso de las efectuadas por Huang \& Walkling (1987), Sandoval (2013), Thanakijsombat (2012) y Zaremba \& Plotnicki (2016) entre muchos otros, pero existen pocos estudios longitudinales que versen sobre el impacto a mediano y largo plazo en la rentabilidad y creación de valor posterior a este tipo de transacciones. Además, los resultados de dichos estudios son contradictorios: algunas investigaciones concluyen en sus resultados que se mejora el desempeño financiero de la compañía adquiriente, otras concluyen que se genera un impacto negativo, mientras que otras indican que no hay cambio significativo en el desempeño posterior de la adquiriente o un desempeño ligeramente significativo.

A pesar de la extensa cantidad de investigaciones relacionadas al tema de FyA, aún existe un desconocimiento de los factores que inciden en el desempeño financiero posterior a las mismas. Algunos autores atribuyen el éxito o fracaso en generar valor a factores como la capacidad de gestión de la administración de la compañía adquiriente, el tipo de adquisición, la forma de pago, a diferencias en la regulación y en las normas contables, a diferencias culturales entre los países, a la experiencia en anteriores adquisiciones, el precio de compra y los motivos de la venta, entre otros factores, dejando la posibilidad de predecir el impacto en la rentabilidad inconcluso. (Haspeslagh \& Jemison ,1991). En opinión de King et al., (2004) a pesar de décadas de estudio, qué impacta desempeño financiero en las FyA continúa sin explicación.

Debido a que los resultados de los estudios que se han realizado no son concluyentes, además de que pocos se han referido a países en vías de desarrollo como México, el propósito del presente estudio será comparar longitudinalmente el desempeño financiero anterior y posterior a fusiones y adquisiciones de empresas mexicanas con la finalidad de determinar si se crea

\section{Desempeño financiero posterior de fusiones y adquisiciones}


o no valor para los accionistas, identificando, además, algunos de los factores que inciden en el éxito o fracaso de las mismas.

El resto de este documento está organizado como sigue: en la segunda sección se presenta una revisión de literatura acerca del tema; en la tercera sección, se exponen las teorías económicas relacionadas con el problema de investigación, así como una descripción de las variables analizadas; la cuarta sección describe la metodología de investigación utilizada. Los resultados se muestran en la quinta sección y, por último, las conclusiones y discusión se presentan en la sexta y última sección.

\section{Revisión de literatura}

El que las empresas crean o no valor posterior la fusión y adquisición ha sido motivo de debate, discusión e investigación desde hace mucho tiempo. Los resultados de estas investigaciones arrojan resultados inconsistentes sobre los cambios en el desempeño posterior de las fusiones y adquisiciones para la compañía adquiriente, así como falta de consenso en identificar los factores que incidieron en dichos resultados, pudiéndose agrupar en tres grandes categorías: a) aquellos que reportan una mejora significativa en el desempeño posterior; b) aquellos que documentan un deterioro significativo y; c) aquellos que no encuentran cambios significativos o muy poco significativos. A continuación, se presentan algunos de ellos.

Dentro del grupo de investigaciones con resultados de éxito para las FyA está el efectuado por Heron \& Lie (2002), en el que estudiaron el efecto del método de pago en el desempeño posterior 859 fusiones y adquisiciones de la American Stock Exchange, NYSE y NASDAQ encontrando que, posterior a las adquisiciones, las adquirentes continúan exhibiendo un desempeño superior en relación con su industria y experimentan niveles significativamente más altos de desempeño operativo.

Resultados similares fueron los encontrados por Rahman \& Limmack (2004) que analizaron el flujo de operación posterior a 94 operaciones de fusiones y adquisiciones en Malasia, encontrando que el rendimiento del flujo de efectivo operativo para las empresas combinadas mejora significativamente después de las adquisiciones gracias tanto a un aumento en la productividad de los activos como a los niveles más altos de flujo de caja operativo generados por unidad de ventas. Así mismo, Carmona et al., (2015) al

\section{Lambretón, T., Villarreal, J., Montemayor, E., Lechuga, J. \& Quiroga, L-}


comparar las razones financieras, así como el precio de mercado posterior de 9 fusiones y adquisiciones efectuadas en la Bolsa Mexicana de Valores (BMV) entre el 2010 y el 2012, encontraron resultados satisfactorios en su rendimiento y crecimiento, concluyendo que las FyA son una estrategia empresarial exitosa para las empresas listadas en la BMV que optan por la generación de valor con las fusiones y adquisiciones. De igual forma, Trivedi (2013) en la investigación efectuada con 30 FyA de compañías que cotizan en las Bolsas de la India, encontró mejoras significativas posteriores en la mayoría de las razones financieras analizadas.

En contraste, resultados contrarios fueron obtenidos en las investigaciones de Dickerson et al., (1997), en el que analizaron el impacto de las FyA en el Reino Unido, sus resultados indican que las adquisiciones tienen un impacto perjudicial en el desempeño de la compañía y que el crecimiento de la compañía a través de la adquisición produce una tasa de rendimiento más baja que el crecimiento a través de la inversión interna. Resultados similares fueron obtenidos por Yeh \& Hoshino (2002) en que examinaron 86 fusiones corporativas entre 1979 y 1994 en Japón, encontrando que las fusiones japonesas no mejoraron la eficiencia de la empresa, e incluso causaron un deterioro en el desempeño operativo. De igual forma, Sharma \& Ho (2002) al analizar 36 FyA en Australia de 1986 a 1991, concluyen que los resultados de rendimiento operativo no muestran ganancias significativas posteriores a la adquisición. Resultados negativos fueron también reportados en las investigaciones de Shan \& Khan (2017) y Gogia (2018).

Por su parte, dentro del grupo de investigaciones en el que se concluye que las FyA no generan un cambio significativo en el desempeño posterior de la adquiriente se encuentra Ghosh (2001), en cuyo estudio se analizaron 315 FyA en Estados Unidos de 1981 a 1995 no encontrando evidencia de mejoras en el rendimiento operativo de las empresas fusionadas después de las adquisiciones. Resultados parecidos obtuvieron Schekaibán et al., (2009) al analizar si las FyA de 1987-2005 de las sociedades anónimas mexicanas han alcanzado sinergias económicas financieras y contables encontrando que, aun y que no se encuentran diferencias significativas, se puede observar una tendencia estable, reorganización y, en el largo plazo, aprovechamiento de las sinergias en cuanto al logro, en cierto grado, de economías de escala y de alcance.

\section{Desempeño financiero posterior de fusiones y adquisiciones}


Resultados con cambios poco significativos fueron encontrados por Powell \& Stark (2005) y por Kruse et al., (2003). El primero de ellos ubicado en Reino Unido durante el período 1985 a 1993, encontrando mejoras modestas en el rendimiento operativo. En el segundo se analizaron 46 FyA de empresas manufactureras que cotizaron en el Tokyo Stock Exchange en el periodo de 1969 a 1992, concluyendo que el efecto a largo plazo del desempeño operativo es positivo pero insignificante.

Debido a que no existe un consenso en los resultados mostrados por investigaciones previas, el presente estudio pretende contribuir al debate sobre las FyA al analizar el desempeño financiero de una muestra de empresas mexicanas que llevaron a cabo fusiones y adquisiciones en los años 2013 y 2014.

\section{Marco teórico}

Los términos fusiones y adquisiciones son frecuentemente utilizados como sinónimos, sin embargo, existen diferencias legales y contables entre ambas. Una fusión ocurre cuando una compañía adquiere la totalidad de los activos netos de otra compañía y ésta última se disuelve, o cuando las dos compañías se disuelven y forman juntas una nueva corporación. Una adquisición, por su parte, ocurre cuando una compañía adquiere la totalidad o solo una parte de las acciones de otra compañía y las dos compañías siguen operando legalmente de manera independiente. Algunas de las razones por las que las compañías efectúan FyA son ahorro en costos, menor riesgo, menores demoras en la operación, para evitar que otra empresa las adquiera y para adquirir activos intangibles, entre otras razones. (Beams et al., 2016)

La teoría económica ha proporcionado muchas posibles razones por las cuales podrían ocurrir fusiones: razones relacionadas con la eficiencia que a menudo involucran economías de escala u otras sinergias; intentos de crear poder de mercado formando monopolios u oligopolios; disciplina de mercado, deseos de la administración de expandirse y aprovechar las oportunidades para la diversificación. Además, las estandarizaciones en el comercio internacional han facilitado que las empresas negocien y creen asociaciones más allá de sus fronteras nacionales para aprovechar al máximo la globalización. (Ubabuko \& Kwaasi Adjei, 2011).

\section{Lambretón, T., Villarreal, J., Montemayor, E., Lechuga, J. \& Quiroga, L-}


Desde el punto de vista de la teoría de la firma, si todas las FyA cumplieran con el objetivo de maximizar las utilidades, los objetivos de los administradores y de los accionistas estarían alineados; sin embargo, si una gran proporción de esas FyA no generan beneficios a largo plazo, entonces significa que las estructuras de gobierno están fallando al no brindar esa alineación. (Gugler et al., 2003).

Las FyA pueden ocurrir por razones económicas o no económicas, el análisis del desempeño posterior es una buena forma de determinar si las FyA fueron o no exitosas. La mayoría de las investigaciones utilizan en la evaluación del desempeño posterior razones de rentabilidad, como son el ROA, ROE y márgenes de utilidad; razones de liquidez y apalancamiento como son la razón circulante, el flujo de operación y la deuda total; así como otras métricas como el total de activo y las ventas totales. Como medidas de creación de valor han sido utilizadas variables como el precio de la acción y el valor de la empresa posterior a la adquisición, entre otras variables. Como ejemplo de este tipo de estudios se tiene el efectuado por Rao-Nicholson et al., (2016) en el que se midió el desempeño posterior de 57 FyA de países asiáticos utilizando el ROA y margen de utilidad, encontrando que las FyA completadas durante períodos de crisis son más exitosas que aquellas efectuadas antes o después de estas, el de Gogia (2018), estudio ubicado en la India que incluyó 20 FyA del sector bancario en el que se midió la razón circulante y el margen de utilidad neta, encontrando un deterioro posterior a las fusiones. Estudios similares en el que se utilizaron razones financieras fueron efectuados por Shah \& Khan (2017) y Rozen (2018), entre otros.

Debido a que los resultados de los estudios previos son contradictorios y no se ha llegado a un consenso acerca de si las FyA generan un mejor desempeño posterior para la compañía adquiriente, en el presente estudio se pretenden atender las siguientes preguntas de investigación:

¿Las fusiones y adquisiciones de las empresas públicas en México generan un mejor desempeño financiero posterior a la adquisición?, y ¿Qué factores influyen en el desempeño financiero posterior a las fusiones y adquisiciones?

En consecuencia, la primera hipótesis alternativa planteada en esta investigación es:

\section{Desempeño financiero posterior de fusiones y adquisiciones}


$H_{1}$ Las fusiones y adquisiciones de las empresas públicas mexicanas generan un cambio significativo en el desempeño financiero posterior de la compañía adquirente.

Se espera, de acuerdo a la revisión de literatura, que las FyA generen un decremento en el desempeño financiero posterior a las FyA. De igual forma, resultados de las investigaciones previas han señalado que hay ciertos factores culturales, económicos, sociales y fiscales, así como factores asociados al proceso llevado a cabo durante las FyA que inciden en el desempeño posterior. En esta investigación se analiza el efecto de 6 de ellos:

Tamaño de empresa adquiriente

El logaritmo natural del activo se ha utilizado en diversas investigaciones como una medida para determinar el tamaño de la empresa. Asimismo, el tamaño de la empresa adquirente puede ser factor de cambio, tanto positivo como negativo, en el desempeño financiero de la adquirente. De acuerdo con Borrás y Belda (2015), el nivel de endeudamiento aumenta a mayor tamaño, sin embargo, a mayor tamaño las empresas generan mayores crecimientos y el costo de insolvencia es menor. Zhao (2019) concluye, después de analizar 437 FyA en China, que el tamaño de la adquiriente tiene un efecto positivo en la rentabilidad a largo plazo. Por el contrario, los resultados de Sedlacek \& Valouch (2018) muestran, después de analizar 201 FyA en República Checa, que no existe relación entre el tamaño de la adquiriente y el éxito en la fusión.

Tipo de pago

Hay dos métodos principales de pago utilizados para financiar operaciones de fusiones y adquisiciones: pagos en efectivo o mediante una emisión de acciones a los accionistas en una proporción acordada, o bien, una mezcla de ambos. Ubabuko \& Kwassi (2011) encontraron que, si el pago es realizado únicamente en efectivo, el índice de liquidez de la adquirente disminuiría, en cambio, si el pago es por medio de acciones, la adquirente podría tener un índice de rentabilidad más bajo. De igual forma, Barbopoulos et al., (2018) encontraron, después de analizar 30,553 acuerdos de FyA en Reino Unido, que el pago en acciones incrementó la rentabilidad posterior a las mismas. Sin embargo, Linn and Switzer (2001) concluyen en su

\section{Lambretón, T., Villarreal, J., Montemayor, E., Lechuga, J. \& Quiroga, L-}


investigación que el cambio en el desempeño financiero de la compañía adquiriente es significativamente mayor para aquellas adquisiciones efectuadas en efectivo que para las que se pagaron con acciones.

En contraste, Heron \& Lie (2001) y Rao-Nicholson et al., (2016) indican que no encontraron evidencia en sus resultados que indiquen que el método de pago transmita información sobre el rendimiento operativo futuro del adquiriente.

\section{Naturaleza de la transacción}

Las fusiones y adquisiciones pueden ocurrir de dos formas, de manera de manera amigable 0 de manera hostil. "La hostilidad generalmente se percibe cuando se hace pública una oferta que es rechazada agresivamente por la empresa a adquirir" Schwert (2000), por lo que una adquisición amigable se da sin presiones de ninguna de las dos partes. Rao-Nicholson et al., (2016) concluyen que la naturaleza amigable de los acuerdos es determinante del desempeño operativo posterior a largo plazo.

En opinión de Sudarsanam \& Mahate, (2006) "En general, tanto los compradores hostiles como los amigables incurren en costos que pueden destruir el valor de sus accionistas. Por lo que es una pregunta empírica qué mecanismo destruye más valor". Su estudio proporciona evidencia del rendimiento superior de creación de valor de los compradores hostiles.

Idioma

El idioma es un factor cultural que puede llegar a ser clave en la creación de valor en las FyA. De acuerdo al estudio de Navio-Marco et al., (2015) las empresas que realizaron fusiones y adquisiciones con empresas de países que hablaban el mismo idioma, creaban mayor valor en el largo plazo. De igual forma, en un estudio llevado a cabo en Estados unidos por Bereskin et al., (2018) concluyen, al analizar 570 acuerdos de FyA, que similitudes culturales como el idioma generan mayores sinergias ocasionando mejor desempeño posterior; resultados similares fueron obtenidos por Boateng et al., (2019) después de analizar 209 FyA en China, al encontrar que la distancia cultural crea un efecto negativo en la rentabilidad en el corto y largo plazo.

\section{Desempeño financiero posterior de fusiones y adquisiciones}


Tipo de industria

La industria a la que pertenezca la empresa adquiriente puede ser un factor que influya en el desempeño posterior a una FyA. Rozen (2018) encontró diferencias en el desempeño posterior dependiendo los diferentes sectores analizados. En contraste, resultados de la investigación efectuada por Mantravadi \& Reddy, (2008) indican que no hubo variaciones significativas en el rendimiento operativo para las diferentes industrias.

Tipo de integración

Existe actualmente tres tipos de categorías para las fusiones y adquisiciones. La primera es la integración vertical que se genera cuando se consolida con otra empresa de otro nivel superior o inferior de la cadena productiva; la segunda es integración horizontal que ocurre cuando se consolida con empresas del mismo giro y nivel en la cadena productiva y, finalmente, el conglomerado, cuando se consolida con una empresa de un giro totalmente diferente a la adquirida. (Beams et al.,2016). Resultados de la investigación efectuada por sectores de Rozen (2018), indican que integraciones horizontales fueron beneficiosas para el sector industrial mas no para el de servicios. La integración vertical fue beneficiosa para el sector de servicios y la integración por conglomerado logró éxito en la integración y sinergias en ambos sectores analizados.

Con el objeto de contestar la segunda pregunta de investigación, la segunda hipótesis alternativa planteada en este estudio tiene la finalidad de identificar si los factores mencionados en los párrafos anteriores inciden en un mejor o peor desempeño financiero posterior.

$\mathrm{H}_{2}$ El desempeño posterior a una fusión o adquisición de la compañía adquiriente está influenciado por: el tamaño de la compañía adquiriente, el tipo de pago, el idioma de la adquirida, la naturaleza de la transacción, el tipo industria, así como por el tipo de integración.

\section{Metodología}

Este estudio de campo mide longitudinalmente el desempeño anterior y posterior a las fusiones y adquisiciones de 24 empresas mexicanas que

\section{Lambretón, T., Villarreal, J., Montemayor, E., Lechuga, J. \& Quiroga, L-}


cotizan en la BMV efectuadas durante el 2013 y 2014 comparando su desempeño financiero cuatro años antes y cuatro años posteriores a las FyA.

El tipo de estudio es correlacional, ya que mide la relación entre dos 0 más variables y probabilístico, ya que se consideran pruebas estadísticas con un nivel de significancia de alfa. Esta investigación utiliza para las pruebas estadísticas el software Statistical Package for the Social Sciences (SPSS) asi como el paquete estadístico de EViews.

La información financiera analizada abarca el período del 2009 al 2018 y se obtuvo de la base de datos de Bloomberg. La función general de Bloomberg empleada para esta investigación es "MA" o "M\&A". Las funciones avanzadas utilizadas fueron: date range, region/country, public/private, deal type, nature of bid, payment type y status. Información complementaria fue obtenida de los informes anuales que presentaron las empresas analizadas en la Bolsa Mexicana de Valores.

Obtención de la muestra

Durante los años 2013 y 2014 se llevaron a cabo 211 transacciones de fusiones y adquisiciones por empresas mexicanas, tanto transfronterizas como domésticas. Para la obtención de la muestra, se eliminaron aquellas transacciones no completadas, las que fueron realizadas por la misma empresa adquiriente; las transacciones que corresponden a un grupo inespecífico de compradores; aquellas en que las empresas adquirentes no son públicas; aquellas con las que no se cuenta información suficiente de los periodos analizados, así como aquellas transacciones en que no se adquirió más del $50 \%$ de participación en la empresa adquirida. La Tabla 1 resume lo anterior.

Tabla 1. Proceso obtención de la muestra

\begin{tabular}{lc}
\hline Descripción & Transacciones \\
\hline Transacciones de FyA en México entre 2013 y 2014 & 211 \\
Menos: & $(14)$ \\
Estado del trato no completado & $(44)$ \\
Tipo de trato diferente a fusión o adquisición & $(20)$ \\
Transacciones efectuados por empresas privadas & $(58)$ \\
No se cuenta con información suficiente de los periodos analizados & $(45)$ \\
Transacciones duplicadas (se repite el adquiriente) & $(06)$ \\
Transacciones que en las que se adquirió participación menor a 50\% & 24 \\
\hline Tamaño de la muestra analizada & \\
\hline
\end{tabular}

Fuente: elaboración propia

\section{Desempeño financiero posterior de fusiones y adquisiciones}


Prueba de la primera hipótesis nula

Para responder la primera pregunta de investigación se efectúa el cómputo de las métricas financieras de rentabilidad, liquidez, apalancamiento y creación de valor incluidas en la Tabla 2 cuatro años antes y cuatro años posteriores a las FyA. Se obtiene el promedio de las mismas antes y después y se evalúa la significancia del cambio en el promedio utilizando las pruebas de T-test cuando los datos siguen una distribución normal y la prueba no paramétrica de Wilcoxon si los datos siguen una distribución no normal. Para determinar la normalidad de los datos es utilizada la prueba de Shapiro-Wilk. La prueba T-test fue utilizada en los estudios de mientras que la prueba de Wilcoxon ha sido utilizada en estudios como el de Sharma \& Ho (2002), Rahman (2004), Powel \& Stark (2005), Rao-Nicholson et al., (2016), entre otros.

Para las pruebas de significancia se rechaza la hipótesis nula que indica que no hay diferencias significativas en las razones financieras antes $y$ después con un nivel de confianza del $90 \%$ cuando el $p$-value $<0.10$.

Para la prueba de normalidad de Shapiro-Wilk, se rechaza la hipótesis nula que indica que los datos están normalmente distribuidos con un nivel de confianza del $95 \%$ cuando el $p$-value $<0.05$. El software utilizado en la prueba de la primera hipótesis nula es SPSS.

Tabla 2. Razones financieras analizadas

\begin{tabular}{lcc}
\hline \multicolumn{1}{c}{ Razón financiera } & \multicolumn{1}{c}{ Cálculo } & $\begin{array}{c}\text { Impacto } \\
\text { esperado }\end{array}$ \\
\hline $\begin{array}{l}\text { Razón circulante } \\
\text { (RC) }\end{array}$ & $\begin{array}{ccc}\text { Activo circulante } \\
\text { Pasivo corto plazo }\end{array}$ & Disminución \\
\hline $\begin{array}{l}\text { Rendimiento sobre el } \\
\text { capital (ROE) }\end{array}$ & Utilidad neta & Disminución \\
\hline $\begin{array}{l}\text { Rendimiento sobre los } \\
\text { activos (ROA) }\end{array}$ & $\begin{array}{c}\text { Utilidad neta } \\
\text { Activo promedio }\end{array}$ & Disminución \\
\hline $\begin{array}{l}\text { Nivel endeudamiento } \\
\text { (NE) }\end{array}$ & $\begin{array}{c}\text { Pasivo total } \\
\text { Activo total }\end{array}$ & Aumento \\
\hline $\begin{array}{l}\text { Utilidad por acción } \\
\text { (UPA) }\end{array}$ & Utilidad neta & Disminución \\
\hline Flujo libre de efectivo & Acciones en circulación & \\
\hline
\end{tabular}

Lambretón, T., Villarreal, J., Montemayor, E., Lechuga, J. \& Quiroga, L- 


\begin{tabular}{|c|c|c|}
\hline (FLE) & $\begin{array}{l}\text { Donde: } \\
\text { FEO = Flujo de efectivo operativo } \\
I A F N=\text { Inversión en activos fijos netos } \\
I A C N=\text { Inversión en activos corrientes netos }\end{array}$ & \\
\hline $\begin{array}{l}\text { Valor Económico } \\
\text { Agregado (VEA) }\end{array}$ & $\begin{array}{l}\text { NOPAT - (CPPC x Capital empleado }) \\
\text { Donde: } \\
\text { NOPAT = Utilidad de operación neta de } \\
\text { impuestos } \\
\text { CPPP = costo promedio ponderado de } \\
\text { capital }\end{array}$ & Disminución \\
\hline
\end{tabular}

Fuente: elaboración propia

Prueba de la segunda hipótesis nula

La segunda hipótesis tiene como finalidad responder a la pregunta de investigación ¿Qué factores influyen en el desempeño financiero posterior a las fusiones y adquisiciones? Para tal efecto, se efectúa un modelo de regresión utilizando el software estadístico de Eviews. El modelo de regresión pretende evaluar la relación que existe entre la variable dependiente " $Y$ " y las variables independientes " $X$ " dentro de las cuales se toman en cuenta los factores que pueden afectar el desempeño financiero posterior a las FyA. Por medio de este modelo se pretende obtener la relación que existe entre ambas variables con la finalidad de determinar cómo se ve afectado el desempeño financiero de las empresas adquirentes con la ocurrencia de los factores analizados en este estudio. Algunos de los estudios previos que han utilizado modelos de regresión lineal son Yeh \& Hoshino (2002), Kruse (2002), Powell (2005), y Rao (2016) entre otros.

Debido a que la muestra es de 24 transacciones y no se cumple con el teorema límite central, se utiliza un modelo de regresión robusta. La finalidad de la prueba de mínimos cuadrados robustos es obtener un método de regresión menos sensible cuando se presentan valores atípicos. En este caso, se utiliza el método de regresión robusto M-estimation, el cual aborda valores atípicos cuando el valor de la variable dependiente difiere sustancialmente de la regla de residuos grandes

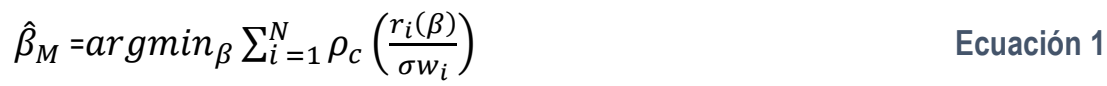

\section{Desempeño financiero posterior de fusiones y adquisiciones}


Donde

$\sigma$ es una medida de la escala de los residuos,

c es una constante arbitraria de giro positivo asociada con la función, $w_{i}$ son los pesos individuales que generalmente se establecen en 1 , pero pueden ser establecidas a:

$$
w_{i=\sqrt{1-X_{I}\left(\left(X^{\prime} X\right)^{-1} X^{\prime}\right.}} \quad \text { Ecuación } 2
$$

La Tabla 3 muestra la operacionalización de las variables utilizadas en la regresión.

Tabla 3. Operacionalización de las variables

\begin{tabular}{ll}
\hline Variables & Descripción \\
\hline \begin{tabular}{l} 
Variable dependiente "Y": \\
\multicolumn{1}{c}{$\mathrm{NE}$}
\end{tabular} & Cambio en el nivel de endeudamiento \\
\hline $\begin{array}{l}\text { Variables Independientes } X: \\
\text { Tipo de pago }\end{array}$ & $1=$ efectivo $0=$ en acciones \\
Naturaleza de la transacción & $1=$ amistoso $0=$ hostil \\
Idioma & $1=$ mismo idioma $0=$ diferente idioma \\
Tipo industria & Industrial, financiera, comunicación, materiales o consumo \\
Tipo de integración & Vertical, horizontal o conglomerado \\
Tamaño de la adquiriente & Logaritmo natural del total de activos en el año de la compra \\
\hline
\end{tabular}

Fuente: elaboración propia

La ecuación general del modelo de regresión utilizada en esta investigación es entonces:

$\Delta Y=\beta_{0}+\beta_{i}$ T.pago $+\beta_{i}$ Naturaleza $+\beta_{i}$ Industria + $\beta_{i}$ T. intergración $+\beta_{i}$ Tamaño $+\beta_{i}$ Idioma $+\varepsilon_{i}$

Ecuación 3

Se toma en consideración el valor de Rw cuadrada ajustada para medir que tanto las variables independientes analizadas explican los cambios en la variable dependiente. El criterio utilizado para cada uno de los factores analizados es la probabilidad del coeficiente, se considera que el factor incide sobre la variable dependiente cuando la probabilidad es menor a 0.10 .

\section{Lambretón, T., Villarreal, J., Montemayor, E., Lechuga, J. \& Quiroga, L-}




\section{Resultados}

En esta sección se presentan los resultados obtenidos en este estudio y que da respuesta a las preguntas de investigación

Prueba de la $1^{\circ}$ hipótesis

La primera hipótesis busca evaluar si los cambios en las razones financieras analizadas posterior a las FyA son o no estadísticamente significativos. Los procesos y cálculos estadísticos que se presentan a continuación se obtuvieron mediante el uso del programa SPSS.

Tabla 4. Estadística descriptiva de las variables analizadas

\begin{tabular}{lccccc}
\hline \multicolumn{1}{c}{ Variables } & Mínimo & Máximo & Mediana & Promedio & Desv.estándar \\
\hline RC ANTE & 0.96 & 34.912 & 1.949 & 4.471 & 7.708 \\
RC POST & 0.54 & 6.312 & 1.83 & 2.119 & 1.359 \\
ROE ANTE & -0.328 & 0.357 & 0.116 & 0.119 & 0.132 \\
ROE POST & -1.366 & 0.186 & 0.102 & 0.038 & 0.304 \\
ROA ANTE & -0.295 & 0.189 & 0.059 & 0.049 & 0.086 \\
ROA POST & -0.791 & 0.117 & 0.028 & 0.007 & 0.173 \\
NE ANTE & 0.02 & 0.719 & 0.187 & 0.227 & 0.179 \\
NE POST & 0.074 & 0.634 & 0.273 & 0.285 & 0.131 \\
UPA ANTE & -0.935 & 21.405 & 1.721 & 3.125 & 4.825 \\
UPA POST & -0.248 & 31.75 & 1.89 & 4.171 & 6.717 \\
FLE ANTE & -635.0 & $87,741.6$ & 932.5 & $7,296.3$ & 19,334 \\
FLE POST & -660.7 & $68,067.3$ & $1,312.8$ & $8,348.5$ & 17,503 \\
VEA ANTE & -43.1 & 9.7 & -0.856 & -2.3 & 10.006 \\
VEA POST & -139.9 & 3.7 & -3.315 & -8.9 & 28.108 \\
\hline FUA
\end{tabular}

Fuente: elaboración propia

Los resultados de las pruebas de normalidad de Shapiro-Wilk muestran que las razones financieras analizadas tienen una distribución no normal, por lo cual se utilizó únicamente la prueba no paramétrica de Wilcoxon para medir la significancia del cambio. La siguiente tabla presenta los cambios en las razones analizadas, así como los resultados de las pruebas de significancia.

Tabla 5. Cambio en las razones analizadas y resultados de la prueba de Wilcoxon

\begin{tabular}{cccccccc}
\hline $\mathbf{n = 2 4}$ & $\begin{array}{c}\text { Promedio } \\
\text { anterior }\end{array}$ & $\begin{array}{c}\text { Promedio } \\
\text { posterior }\end{array}$ & Cambio & $\begin{array}{c}\text { Cambio } \\
\text { porcentual }\end{array}$ & $\begin{array}{c}\text { Z- } \\
\text { value }\end{array}$ & $\begin{array}{c}\text { P- } \\
\text { value }\end{array}$ & Significancia \\
\cline { 2 - 6 } & $\mathbf{A}$ & $\mathbf{B}$ & $\mathbf{B}-\mathbf{A}$ & $\mathbf{( B - A ) / A}$ & & \\
\hline ROA & $4.90 \%$ & $0.70 \%$ & $-4.20 \%$ & $-85.96 \%$ & -2 & 0.046 & $* *$
\end{tabular}

\section{Desempeño financiero posterior de fusiones y adquisiciones}




$\begin{array}{lccccccc}\text { ROE } & 11.90 \% & 3.80 \% & -8.10 \% & -67.73 \% & -1.943 & 0.052 & * \\ \text { UPA } & 3.125 & 4.171 & 1.045 & 33.45 \% & -1.571 & 0.116 & \\ \text { RC } & 4.471 & 2.119 & -2.352 & -52.60 \% & -2.451 & 0.014 & * \\ \text { NE } & 22.70 \% & 28.50 \% & 5.80 \% & 25.64 \% & -3.029 & 0.002 & * \\ \text { FLE } & 7,296.34 & 8.348 .46 & 1,052.12 & 14.42 \% & -2.143 & 0.032 & * \\ \text { VEA } & -2.344 & -8.974 & -6.629 & -282.77 \% & -2.2 & 0.028 & \text { ** }\end{array}$

Fuente: elaboración propia con base en resultados de prueba Wilcoxon con software SPSS. Nota: significativo con nivel confianza $99 \%{ }^{* * *}$; significativo con nivel de confianza $95 \%{ }^{* *}$, significativo con nivel de confianza del $90 \%$ *; si p-value mayor a 0.10 no significativo.

En cuanto la rentabilidad, los cambios tanto en el ROA como en el ROE fueron desfavorables y estadísticamente significativos con una disminución en promedio en el ROA de $4.90 \%$ a $0.70 \%$ lo que representa una disminución del $85.96 \%$, en promedio, el ROE disminuyó de $11.90 \%$ a $3.8 \%$ es decir un decremento del $67.73 \%$. Por su parte, la utilidad por acción, aunque presenta un incremento del $33.45 \%$, dicho cambio resultó no ser estadísticamente significativo.

En relación a liquidez y endeudamiento, los resultados fueron no favorables debido a que la razón circulante tuvo un cambio estadísticamente significativo al disminuir de 4.471 a 2.119 veces, lo que representa una disminución del $52.60 \%$. De igual forma, el nivel de endeudamiento tuvo el cambio más significativo al incrementarse en promedio del $22.70 \%$ al $28.50 \%$, es decir un incremento en el nivel de endeudamiento del $25.64 \%$.

En cuanto a razones financieras de creación de valor, los resultados son contradictorios, por una parte, el flujo libre de efectivo se incrementó en promedio un $14.42 \%$, mientras que el valor económico agregado presenta una diminución de $282.77 \%$.

Por lo tanto, las pruebas de significancia realizadas a las razones financieras analizadas permiten rechazar la hipótesis nula y aceptar la hipótesis planteada en esta investigación para todas las variables con excepción de la utilidad por acción que no presentó cambios estadísticamente significativos. La siguiente tabla resumen lo anterior.

Tabla 6. Resumen resultados prueba hipótesis \#1

\begin{tabular}{ll}
\hline Variables & Prueba hipótesis nula \#1 \\
\hline Razón circulante & Se rechaza la hipótesis nula \\
Rendimiento sobre activos & Se rechaza la hipótesis nula \\
Rendimiento sobre el capital & Se rechaza la hipótesis nula
\end{tabular}

Lambretón, T., Villarreal, J., Montemayor, E., Lechuga, J. \& Quiroga, L- 


\begin{tabular}{ll} 
Nivel de endeudamiento & Se rechaza la hipótesis nula \\
Utilidad por acción & Se acepta la hipótesis nula \\
Flujo libre de efectivo & Se rechaza la hipótesis nula \\
Valor económico agregado & Se rechaza la hipótesis nula \\
\hline
\end{tabular}

Fuente: Elaboración propia con base a resultados prueba Wilcoxon

Prueba de la $2^{\circ}$ hipótesis

El modelo de regresión utilizado para evaluar qué factores inciden en un mejor o peor desempeño posterior consideró la razón financiera cambio en el nivel de endeudamiento ya que es la que mayor significancia presentó al medir su cambio posterior a las FyA:

$$
\begin{gathered}
\Delta N E=\beta_{0}+\beta_{i} \text { T.pago }+\beta_{i} \text { Naturaleza }+\beta_{i} \text { Industria }+ \\
\beta_{i} \text { T.intergración }+\beta_{i} \text { Tamaño }+\beta_{i} \text { Idioma }+\varepsilon_{i}
\end{gathered}
$$

Ecuación 4

Para probar la relación entre las variables se efectuó, en primera instancia, una regresión de mínimos cuadrados ordinaria. El tipo de industria usado como referencia fue el financiero y en tipo de integración utilizado el horizontal. Los resultados fueron corregidos con la corrección para autocorrelación de Newey-West ya que la prueba de correlación de los residuos de Breush-Godfrey no fue concluyente. El resultado de la regresión lineal se muestra a continuación.

Tabla 7. Regresión mínimos cuadrados para cambio en el nivel de endeudamiento

\begin{tabular}{lrrrr}
\hline Variable & Coeficiente & Error estándar & Estadístico Z & Probabilidad \\
\hline $\mathrm{C}$ & 30.2951 & 17.0095 & 1.7811 & 0.1052 \\
Idioma & -8.9599 & 6.1890 & -1.4477 & 0.1783 \\
Tamaño & -2.9714 & 1.3393 & -2.2187 & 0.0508 \\
Naturaleza & -0.3887 & 4.6275 & -0.0840 & 0.9347 \\
Integración conglomerado & 7.0498 & 5.2004 & 1.3556 & 0.2050 \\
Integración vertical & -8.8828 & 4.7174 & -1.8830 & 0.0891 \\
Tipo de pago & 23.3711 & 7.4708 & 3.1283 & 0.0107 \\
Materiales & -3.2936 & 6.2640 & -0.5258 & 0.6105 \\
Consumo & -7.5484 & 9.2633 & -0.8149 & 0.4341 \\
Comunicaciones & -15.9816 & 7.2211 & -2.2132 & 0.0513 \\
Industrial & -17.7449 & 8.0212 & -2.2122 & 0.0514 \\
\hline R-cuadrada & 0.6448 & R-cuadrada ajustada \\
Estadístico F & 1.8150 & Probabilidad (estadístico F) & 0.2895 \\
& \multicolumn{4}{l}{0.1807}
\end{tabular}

\section{Desempeño financiero posterior de fusiones y adquisiciones}


Durbin-Watson

0.9909

Fuente: elaboración propia con base a resultados Least-Squares de Eviews

Debido a que en las pruebas efectuadas a la regresión de mínimos cuadrados se detectan problemas de heteroscedasticidad, hay observaciones atípicas (outliers), además de que se encontró al efectuar la prueba de JarqueBera (probabilidad de 0.066) que los errores no se distribuyen normalmente, se consideró que modelo de regresión de mínimos cuadrados no es adecuado al no ser confiable. Derivado de lo anterior, aunado a que el número de observaciones en la muestra no cumple con el teorema del límite central, se decidió utilizar un método de Regresión Robusta que elimine los problemas anteriormente mencionados y que de mayor confiabilidad a los resultados. La Tabla 8 muestra los resultados de la regresión robusta para cambio en el nivel de endeudamiento.

Tabla 8. Resultados regresión robusta cambio NE

\begin{tabular}{lrrrr}
\hline Variable & Coeficiente & Error estándar & Estadístico Z & Probabilidad \\
\hline C & 36.0202 & 17.3656 & 2.0742 & 0.0381 \\
Idioma & -10.2326 & 4.2491 & -2.4082 & 0.0160 \\
Tamaño & -3.3189 & 1.2057 & -2.7526 & 0.0059 \\
Naturaleza & -1.4017 & 4.5173 & -0.3103 & 0.7563 \\
Integración conglomerado & 11.7898 & 4.5534 & 2.5893 & 0.0096 \\
Integración vertical & -5.0979 & 5.7977 & -0.8793 & 0.3792 \\
Tipo de pago & 23.6655 & 10.6860 & 2.2146 & 0.0268 \\
Materiales & -4.1629 & 8.6616 & -0.4806 & 0.6308 \\
Consumo & -12.6344 & 8.9860 & -1.4060 & 0.1597 \\
Comunicaciones & -16.1936 & 10.5915 & -1.5289 & 0.1263 \\
Industrial & -18.6362 & 9.9724 & -1.8688 & 0.0617 \\
\hline Estadísticos Robustos & \multicolumn{4}{|c}{} \\
R-cuadrada & 0.5162 & R-cuadrada ajustada \\
Rw-cuadrada & 0.8467 & Rw-cuadrada ajustada & 0.0323 \\
Estadístico Rn-cuadrado & 25.7211 & Probabilidad (Rn-cuadrado) & 0.8467 \\
\hline
\end{tabular}

Fuente: elaboración propia con base a resultados ROBUSTLS de Eviews

La prueba de regresión robusta para la razón financiera cambio en el nivel de endeudamiento muestra, considerando el valor de Rw-cuadrada ajustada, que los factores considerados en el presente estudio explican en un

\section{Lambretón, T., Villarreal, J., Montemayor, E., Lechuga, J. \& Quiroga, L-}


$84.67 \%$ el cambio del nivel de endeudamiento. El estadístico Rn-cuadrado de 25.72 y su correspondiente probabilidad de 0.0041 indica un fuerte rechazo a la hipótesis nula que indica que todos los coeficientes no interceptados son iguales a cero. En cuanto a los coeficientes de la regresión, a continuación, se presenta la interpretación para cada uno de ellos.

Para el factor tipo de pago, el coeficiente positivo indica, con un nivel de confianza del $95 \%$, que un pago en efectivo incrementa en 23.67 puntos porcentuales el nivel de endeudamiento. Estos resultados son consistentes con los de Ubabuko \& Kwassi (2011) en el que concluyen que, si el pago es realizado únicamente en efectivo, el índice de liquidez de la adquirente disminuye.

El signo negativo del coeficiente para el factor idioma indica, con un nivel de confianza del $95 \%$, que si el idioma de la adquirida es el mismo que la adquiriente ocasiona una diminución en el nivel de endeudamiento de 10.23 puntos porcentuales, lo que es consistente con los resultados de Navio-Marco et al., (2015) quienes, aunque no midieron nivel de endeudamiento, consideran que un mismo idioma ocasionaba que las empresas crearan mayor valor en el largo plazo.

En cuanto al factor tamaño, el coeficiente con signo negativo indica, con un nivel de confianza del $99 \%$ que, por cada unidad de cambio en el tamaño de la adquiriente, la deuda disminuye en 3.31 puntos porcentuales, contrario a lo indicado por Borrás y Belda (2015), al sostener que el nivel de endeudamiento aumenta a mayor tamaño y a lo concluido por Sedlacek, (2018) de que no hay relación entre el tamaño y el éxito de las FyA.

El factor naturaleza resulta no ser estadísticamente significativo, por lo que se concluye que el tipo de adquisición amigable $u$ hostil no es un factor determinante para el cambio en el nivel de endeudamiento posterior a las FyA, contrario a Rao-Nicholson et al., (2016) en cuyo estudio concluyen que la naturaleza amigable de los acuerdos es determinante del desempeño operativo posterior a largo plazo.

En relación al factor tipo de integración, los resultados de la regresión indican con un nivel de confianza del $99 \%$ que, si el tipo de integración es de conglomerado, aumenta en 11.78 puntos porcentuales el nivel de endeudamiento. La integración horizontal al ser utilizada como referencia, tendría una influencia menor que conglomerado en cuanto al cambio en el nivel de endeudamiento. La integración vertical sería considerada como la que

\section{Desempeño financiero posterior de fusiones y adquisiciones}


menos efecto tiene sobre el nivel de endeudamiento, sin embargo, no presenta una probabilidad significativa, por lo que no se puede concluir para este tipo de integración. Estos resultados son lo que se esperaba ya que, al adquirir una empresa de un giro completamente diferente, no se logran todas las ventajas de sinergia en costos que lograría mejoras en el desempeño financiero de la adquiriente.

Para el factor industria a la que pertenezca la compañía adquiriente, las probabilidades resultan ser no significativas para los sectores materiales, consumo y comunicaciones y solo poco significativo para el sector industrial con un nivel de confianza del $90 \%$, por lo que no se puede concluir que el sector al que pertenezca la compañía adquiriente sea determinante del desempeño posterior de las FyA. Este resultado es similar al de Mantravadi y Reddy (2008), quienes concluyeron en su investigación que no hubo variaciones significativas en el rendimiento operativo para las diferentes industrias y contrario Rozen (2018) quien si encontró diferencias en el desempeño posterior dependiendo los diferentes sectores analizados.

Derivado de lo anterior, se rechaza parcialmente la segunda hipótesis nula para los factores idioma, tamaño, tipo de pago y tipo de integración, ya que se determinó que influyeron en el cambio en el nivel de endeudamiento posterior y se acepta la hipótesis nula para los factores sector y naturaleza de la transacción.

\section{Discusión}

Las fusiones y adquisiciones han demostrado ser una estrategia popular para lograr el crecimiento y la diversidad de las operaciones de las empresas, sin embargo, la tasa de FyA fallidas indica que los tomadores de decisiones no tienen una compresión profunda de todas las variables que involucra una implementación exitosa, ya que se requiere, además de una buena planificación financiera previa a la adquisición, un proceso de implementación que permita la integración y tenga congruencia para las dos compañías, de forma tal que lo que está en papel se logre poner en la práctica.

Al examinar el efecto que las fusiones y adquisiciones generaron en el desempeño financiero posterior en un grupo de empresas mexicanas se logró cumplir el objetivo planteado en esta investigación, ya que se identificaron algunos de los factores que incidieron en fracaso de las mismas.

\section{Lambretón, T., Villarreal, J., Montemayor, E., Lechuga, J. \& Quiroga, L-}


Los resultados obtenidos no muestran evidencia de que en México las FyA tengan un efecto beneficioso en el desempeño posterior de las compañías adquirientes ya que, en la mayoría de las razones financieras analizadas, se generó un impacto negativo tanto en el desempeño financiero como en la creación de valor. Se registran disminuciones significativas en las métricas de rentabilidad de ROA y ROE; así como un decremento en las razones de liquidez al disminuir la razón circulante y aumentar los niveles de apalancamiento. En cuanto a creación de valor, se encontraron resultados contradictorios, ya que se generó una disminución significativa en el valor económico agregado mientras que el flujo libre de efectivo tuvo una mejora posterior a las FyA.

Los resultados obtenidos en esta investigación son consistentes con los obtenidos por Gogia (2018) quienes concluyen que el desempeño operativo tiende a declinar en los tres años posteriores a las FyA. De igual forma, coinciden con los resultados de Shah \& Khan (2017) quienes encontraron un deterioro en los bancos adquirientes que analizaron en el período posterior a las fusiones.

Por otra parte, el análisis de regresión robusta muestra que los factores como el idioma y tamaño tienen un efecto significativamente positivo en el cambio del nivel de endeudamiento. De forma opuesta, el que el tipo de integración sea conglomerado influye significativamente en el cambio de deuda de forma negativa, al igual que cuando el tipo de pago es en efectivo. En cuanto al factor sector, no se encontraron diferencias significativas en el desempeño posterior entre los diferentes sectores analizados. De forma similar, los resultados indican que la naturaleza de la transacción no fue un factor determinante para el éxito o fracaso de las fusiones y adquisiciones en México.

Este estudio ayuda a comprender algunos de los factores que pudieron haber contribuido al fracaso de las FyA en México en el corto plazo, sin embargo, estos resultados conducen a más preguntas tendientes a identificar las causas de las fallas y a encontrar la forma de prevenirlas. Un examen más profundo de las razones de la alta tasa de fracaso permitiría a las empresas comprender e implementar medidas correctivas apropiadas para aquellas empresas que buscan, a través de las FyA, lograr un crecimiento y creación de valor para sus accionistas. Aunado a lo anterior, el tamaño de la muestra de este estudio no permite generalizar los resultados, por lo que se

\section{Desempeño financiero posterior de fusiones y adquisiciones}


recomienda, para futuras investigaciones, ampliar tanto el período de estudio como el tamaño de la muestra.

\section{Referencias}

Andrade, G., Mitchell, M., \& Stafford, E. (2001). New evidence and perspectives on mergers. Journal of economic perspectives, 15(2), 103-120.

Asquith, P., Bruner, R. F., \& Mullins Jr, D. W. (1983). The gains to bidding firms from merger. Journal of financial economics, 11(1-4), 121-139.

Barbopoulos, L. G., Paudyal, K., \& Sudarsanam, S. (2018). Earnout Deals: Method of Initial Payment and Acquirers' Gains. European Financial Management, 24(5), 792-828. doi: 10.1111/eufm.12135

Beams, F. A., Anthony, J. H., Bettinghaus, B., \& Smith, K. A. (2016). Advanced accounting. Pearson Education Limited 2018.

Bereskin, F., Byun, S. K., Officer, M. S., \& Oh, J.-M. (2018). The Effect of Cultural Similarity on Mergers and Acquisitions: Evidence from Corporate Social Responsibility. Journal of Financial \& Quantitative Analysis, 53(5), 1995-2039.

Boateng, A., Du, M., Bi, X., \& Lodorfos, G. (2019). Cultural Distance and Value Creation of Cross-Border M\&A: The Moderating Role of Acquirer Characteristics. International Review of Financial Analysis, 63, 285-295.

Borrás, B. C., \& Belda, P. R. (2015). Determinantes de la estructura financiera de las empresas españolas. Estudios de economía aplicada, 33(2), 513-531.

Carmona-Vega, E. G. C., Rangel, K. R. L., \& Pérez-lñigo, J. M. (2015). Fusiones y adquisiciones: emisoras que cotizan en la Bolsa Mexicana de Valores como estrategia para la generación de valor. Revista del Centro de Investigación. Universidad La Salle, 11(44), 31-68.

Damodaran, A. (2005). The value of synergy. Stern school of Business, 132-146.

Dickerson, A. P., Gibson, H. D., \& Tsakalotos, E. (1997). The impact of acquisitions on company performance: Evidence from a large panel of UK firms. Oxford economic papers, 49(3), 344-361.

Ghosh, A. (2001). ¿Does operating performance really improve following corporate acquisitions? Journal of corporate finance, 7(2), 151-178.

Gogia, N. (2018). A Study of Indian Private and Public Sector Banks with Special Reference to Impact of Post-Merger and Acquisition Venture on Liquidity and Profitability. Adhyayan: A Journal of Management Sciences, 8(2), 13-18. https://doi.org/10.21567/adhyayan.v8i1.14501

Gugler, K., Mueller, D. C., Yurtoglu, B. B., \& Zulehner, C. (2003). The effects of mergers: an international comparison. International journal of industrial organization, 21(5), 625653.

Haspeslagh, P. C., \& Jemison, D. B. (1991). Making acquisitions work. Institut Europe'en d'Administration des Affaires (INSEAD), 77-Fontainebleau (FR)

Heron, R., \& Lie, E. (2002). Operating performance and the method of payment in takeovers. Journal of Financial and quantitative analysis, 37(1), 137-155.

\section{Lambretón, T., Villarreal, J., Montemayor, E., Lechuga, J. \& Quiroga, L-}


Hofstede, G. (2011). Dimensionalizing cultures: The Hofstede model in context. Online readings in psychology and culture, 2(1), 8 https://doi.org/10.1017/S0022109018000716

Huang, Y. S., \& Walkling, R. A. (1987). Target abnormal returns associated with acquisition announcements: Payment, acquisition form, and managerial resistance. Journal of financial economics, 19(2), 329-349.

King, D. R., Dalton, D. R., Daily, C. M., \& Covin, J. G. (2004). Meta-analyses of post-acquisition performance: Indications of unidentified moderators. Strategic management journal, 25(2), 187-200.

Kruse, T. A., Park, H. Y., Park, K., \& Suzuki, K. (2003). The value of corporate diversification: evidence from post-merger performance in Japan.

Linn, S. C., \& Switzer, J. A. (2001). ¿Are cash acquisitions associated with better postcombination operating performance than stock acquisitions? Journal of Banking \& Finance, 25(6), 1113-1138.

Mantravadi, D. P., \& Reddy, A. V. (2008). Post-merger performance of acquiring firms from different industries in India. International Research Journal of Finance and Economics, (22).

Navío-Marco, J., Solórzano-García, M., Matilla-García, M., \& Urueña, A. (2016). Language as a key factor of long-term value creation in mergers and acquisitions in the telecommunications sector. Telecommunications Policy, 40(10-11), 1052-1063.

Powell, R. G., \& Stark, A. W. (2005). Does operating performance increase post-takeover for UK takeovers? A comparison of performance measures and benchmarks. Journal of Corporate Finance, 11(1-2), 293-317.

Rahman, R. A., \& Limmack, R. J. (2004). Corporate acquisitions and the operating performance of Malaysian companies. Journal of Business Finance \& Accounting, 31(3-4), 359-400.

Rao-Nicholson, R., Salaber, J., \& Cao, T. H. (2016). Long-term performance of mergers and acquisitions in ASEAN countries. Research in International Business and Finance, 36, 373-387.

Rozen-BakheR, Z. Comparison of merger and acquisition (M\&A) success in horizontal, vertical and conglomerate M\&As: industry sector vs. services sector. Service Industries Journal, v. 38, n. 7/8, p. 492-518, 2018. DOI 10.1080/02642069.2017.1405938.

Sandoval, E. (2013). Efecto sobre la riqueza de los accionistas de D\&S y empresas del sector retail chileno producto del anuncio de oferta pública de adquisición de sus acciones. Revista Internacional Administración \& Finanzas, 6(2), 1-13.

Schekaibán, N. F. C., Labatut-Serer, G., \& Apaiisi-Caudelli, J. A. (2009). Fusiones y adquisiciones de empresas en México: Análisis económico-financiero y fiscal. CienciaUAT, 4(1), 64-67.

Schwert, G. W. (2000). Hostility in takeovers: in the eyes of the beholder? The Journal of Finance, 55(6), 2599-2640.

Seal \&Associates(2017) Reporte anual Fusiones y Adquisiciones en México. Recuperado http://mnamexico.com/wp-content/uploads/2018/01/Fusiones-y-Adquisiciones-Mexico 2017.pdf

\section{Desempeño financiero posterior de fusiones y adquisiciones}


Sedlacek, J., \& Valouch, P. (2018). Mergers of Trading Companies and Causes of Their failure. Engineering Economics, 29(4), 397-404.

Shah, B. A., \& Khan, N. (2017). Impacts of Mergers and Acquisitions on Acquirer Banks' Performance. Australasian Accounting Business \& Finance Journal, 11(3), 30-54. https://doi.org/10.14453/aabfj.v11i3.4

Sharma, D. S., \& Ho, J. (2002). The impact of acquisitions on operating performance: Some Australian evidence. Journal of Business Finance \& Accounting, 29(1-2), 155-200.

Sudarsanam, S., \& Mahate, A. A. (2006). Are friendly acquisitions too bad for shareholders and managers? Long-term value creation and top management turnover in hostile and friendly acquirers. British Journal of Management, 17(S1), S7-S30.

Thanakijsombat, T. (2012). Gains from Acquisitions and Sources of Financing: The Case of Cross-border Acquisitions by Emerging-market Multinationals. Southern New Hampshire University.

Trivedi, J. C. (2013). "A Study on Pre and Post Performance Evaluation of Merger and Acquisition of top Companies of BSE and NSE". SIES Journal of Management, 9(2).

Ubabuko, K., \& Adjei, E. K. (2011). The Consequences of Post-Merger \& Acquisition Performance in Listed and Non-Listed Company in Sweden: A Case Study for AstraZeneca AB, Cybercom AB, Grant Thornton Sweden AB and PayEx. Cybercom $A B$, Grant Thornton Sweden $A B$ and PayEx (June 10, 2011).

Yeh, T. M., \& Hoshino, Y. (2002). Productivity and operating performance of Japanese merging firms: Keiretsu-related and independent mergers. Japan and the World Economy, 14(3), 347-366.

Zaremba, A., \& Płotnicki, M. (2016). Mergers and acquisitions: Evidence on postannouncement performance from CEE stock markets. Journal of Business Economics and Management, 17(2), 251-266.

Zhao, X., Ma, H., \& Hao, T. (2019). Acquirer size, political connections and mergers and acquisitions performance. Studies in Economics and Finance. v. 36, n. 2, p. 311-332, 2019. DOI 10.1108/SEF-05-2017-0112

\section{Lambretón, T., Villarreal, J., Montemayor, E., Lechuga, J. \& Quiroga, L-}

